\title{
A Trans-acting Factor May Modify Age at Onset in Familial Amyloid Polyneuropathy ATTRV30M in Portugal
}

\author{
Miguel Alves-Ferreira ${ }^{1,2} \&$ Teresa Coelho ${ }^{3} \&$ Diana Santos $^{1,2} \&$ Jorge Sequeiros ${ }^{1,2} \&$ \\ Isabel Alonso ${ }^{1,2} \&$ Alda Sousa $^{1,2} \&$ Carolina Lemos ${ }^{1,2,4}$
}

Received: 31 December 2016 / Accepted: 2 May 2017 / Published online: 19 May 2017

\begin{abstract}
Although all familial amyloid polyneuropathy (FAP) ATTRV30M patients carry the same causative mutation, early $(<40)$ and late-onset forms $(\geq 50$ years) of FAP may coexist in the same family. However, this variability in age at onset is still unexplained. To identify modifiers closely linked to the TTR locus that may in part be associated with age at onset of FAP ATTRV30M, in particular in a group of very early-onset patients ( $\leq 30$ years) when compared with lateonset individuals. A clinical genetic study at a referral center comprising a sample of 910 Portuguese individuals includes 589 Val30Met carriers, 102 spouses, and 189 controls from the general population. Haplotype analysis was performed, using eight intragenic single nucleotide polymorphisms (SNPs) at the TTR locus. We compared haplotypes frequency in FAP samples and controls and in parent-offspring pairs using appropriated statistical analysis. Haplotype A was the most common in the general population. Noteworthy, haplotype $\mathrm{C}$ was more frequent in early-onset $(<40)$ than in lateonset patients $(\geq 50$ years $)(p=0.012)$. When comparing allelic frequencies of each SNP within haplotype $\mathrm{C}$ between Bvery early^ ( $\leq 30$ years) and late-onset ( $\geq 50$ years) cases, the A allele of rs72922947 was associated with an earlier onset
\end{abstract}

$*$ Carolina Lemos clclemos@ibmc.up.pt

1 UnIGENe, IBMC-Institute for Molecular and Cell Biology; Institute for Research and Innovation in Health Sciences (i3S), University of Porto, 4200-135 Porto, Portugal

2 ICBAS-InstitutoCiências Biomédicas Abel Salazar, Universidade do Porto, 4050-313 Porto, Portugal

3 Unidade Corino de Andrade (UCA), Centro Hospitalar do Porto (CHP), Largo Prof. Abel Salazar, 4050 Porto, Portugal

4 UnIGENe, IBMC-i3S, Rua Jorge Viterbo Ferreira, 228, 4050-313 Porto, Portugal
( $p=0.009)$; this remained significant after a permutationbased correction. Also, the heterozygous genotype (GA) for this SNP was associated with a decrease in mean age at onset of 8.6 years $(p=0.014)$. We found a more common haplotype (A) linked to the Val30Met variant and a possible modulatory trans effect on age at onset. These findings may lead to potential therapeutical targets.

Keywords Familial amyloid polyneuropathy (FAP) .

Transthyretin-relatedamyloidosis $\cdot$ Transeffect $\cdot$ Transthyretin (TTR) · Age at onset · Haplotype

\section{Introduction}

Familial amyloid polyneuropathy (FAP [MIM: 105210]) ATTRV30M is a severe autosomal dominant (AD) systemic amyloidosis, first described by Andrade in Portugal in 1952 [1] as occurring mainly between the ages of 25 to 35 years. It is due to a sequence variant in the transthyretin (TTR [MIM: 176300]) gene (chr18q12.1); more than 100 disease-causing variants have been found in the TTR gene, but Val30Met

(Val50Met, following HGVS) is by far the commonest, including in Portugal [2].

Awider variability in age at onset $(\mathrm{AO})$ has been uncovered along the years, including among Portuguese patients (1982 years) [3, 4]. Remarkable differences in the distribution of AO were found among FAP clusters associated to Val30Met (Portugal: 35.1; Brazil: 34.5; Japan: 33.8; Sweden: 56.7; Balearic Islands: 45.7 years., in mean AO) [5, 3, 6-8]. A large variation is found, nevertheless, within a single geographical area and even within the same family.

Early ( $\mathrm{AO}<40$ years) and late-onset cases $(\mathrm{AO} \geq 50)$ often coexist in the same family, with offspring often showing an earlier AO than their affected parent (anticipation). We have 
shown that anticipation is a true biological phenomenon in FAP Portuguese families, some parent-offspring pairs showing $\geq 10$ years of difference in AO [9].

This variability in AO is still unexplained. One of the possible hypotheses is the existence of genetic modifiers within or close to the TTR locus. A cis-acting effect of closely linked TTR gene modifiers has been for long postulated by our group $[10,4]$.

Additionally, Coelho et al. found that $40 \%$ of the probands had no affected parent at the time of diagnosis: onset in the transmitting parent happened only after the proband's or the transmitting parent may even die at late age without disease symptoms [11]. These probands, however, had a mean later AO (45.1 years) than those with one affected parent (31.2 years) [11]. In addition, late-onset cases ( $\geq 50$ years) often had offspring with very early onset ( $\leq 30$ years), but the reverse was never found: that Bprotection $\wedge$ may be lost in just one generation [9]. This raised the hypothesis of a closely linked modifier, and shows we need to concentrate in the differences within the same population and, whenever possible, the same family.

Previous studies with haplotypes have been performed in FAP but most of them focused only on the origin of the mutation [12, 13]. Only one major disease haplotype has been found among Portuguese patients [14, 15]. Soares et al. [15] found that onset could be modulated by a region downstream the TTR locus in the non-carrier chromosome. Our aimnow was to identify genetic modifiers within or closely linked to the $T T R$ gene.

\section{Subjects and Methods}

\section{Subjects}

Unidade Corino de Andrade-UCA (Hospital Santo António, Centro Hospitalar do Porto: HSA, CHP) has the largest dataset of FAP ATTRV30M worldwide, with registries that have been collected and clinically characterized over 75 years (since Andrade's first observation, in 1939), by the same team of neurologists. This dataset has invaluable familial and clinical information (including information on AO), from which we collected 721 samples, belonging to 155 families with DNA available from at least 2 relatives; from this group, 589 were Val30Met carriers while 132 were healthy individuals (spouses and non-carrier siblings) from the same families, that helped in haplotype's reconstruction (Table 1). All spouses were genotyped for Val30Met mutation and found to be non-carriers. Additionally, we evaluated 101 parentoffspring transmissions for haplotypes construction and allelic transmission. We also used 189 controls (63 trios) non-V30M from the general population, previously ascertained at the Centro de Genética Preditiva e Preventiva (CGPP, IBMCi3S) biobank, authorized by CNPD (National Commission for Data Protection). Written informed consent was obtained for all participants and the Ethics Committee of HSA, CHP, approved this study. Late-asymptomatic carriers ( $\geq 50$ years) were included in the late-onset sample in order to increase the sample power.

\section{Methods}

\section{Definition of Age at Onset (AO) and AO Groups}

AO was defined, as before [3], as the beginning of the first symptoms (either sensitive or dysautonomic), coincident with an abnormal neurological or neurophysiological observation, reported by the neurologist. Although AO is described in the literature as early $(<40)$ and as late onset $(\geq 50$ years), in addition, we introduced here a Bvery early $\wedge$ sub-group for patients with onset $\leq 30$ years in order to further explore this high-risk group as described in [9].

\section{Genomic DNA Extraction}

Genomic DNAwas isolated from peripheral blood leucocytes, using a standard salting-out method [16]; or from saliva, using ORAGENE® kits, according to manufacturer's instructions (DNA Genotek Inc. Kanata, ON, Canada).

\section{Selection of Single Nucleotide Polymorphisms (SNPs)}

We selected SNPs based on data available for the Caucasian population in the International HapMap project (Release 24, November 2008, on NCBI B36 assembly, dbSNP build 126) and the 1000 genomes database. Eight SNPs were selected due to their short genetic distance to Val30Met mutation and genotyped, from which four were tagging SNPs, selected using Haploview v.4.1 [17], with the following parameters: $r^{2}>0.8$ (as a measure of linkage disequilibrium, LD) and a
Table 1 Age at onset, gender distribution, and disease status in the study group

\begin{tabular}{lrlllllll}
\hline Healthy individuals & \multicolumn{7}{c}{ Val30Met carriers } \\
\hline Spouses & 102 & & $\leq 30$ & $31-40$ & $41-49$ & $\geq 50$ & Assint $<50$ & Assint $\geq 50$ \\
Non-carriers siblings & 30 & Male & 72 & 51 & 12 & 53 & 60 & 12 \\
Male & 69 & Female & 35 & 80 & 33 & 60 & 100 & 21 \\
Female & 63 & Total & 107 & 131 & 45 & 113 & 160 & 33 \\
\hline
\end{tabular}


minor allele frequency $(\mathrm{MAF}) \geq 0.10$. These parameters allowed selecting tagging SNPs covering a total of $57 \mathrm{~kb}$ of the common variation present in the TTR gene (Table 2). The other four SNPs were selected according to previous descriptions of Soares et al. [15] and Li et al. [18].

\section{Genotyping}

For the selected SNPs, PCR primers were designed using the software Primer3; dimer and hairpin formation were excluded using Autodimer. Primer sequences are available upon request.

The multiplex PCR reaction was performed using the Multiplex PCR Master Mix (Qiagen, Hilden, Germany), and experiments conducted according to the standard pro- tocol. After PCR, unincorporated deoxynucleotides (dNTPs) were removed with Exo SAP-IT ( USB Corporation, Cleveland, $\mathrm{OH}$ ), as recommended by the manufacturer. The SNaPshot technique was used to perform allelic discrimination of SNPs. The probes were also tested in AutoDimer software. Probes sequences are also available upon request. The SNaPshot (Applied Biosystems, Carlsbad, CA) reaction was performed according to manufacturer's instructions. After the minisequencing reaction, the excess of ddNTPs was removed by incubating the SNaPshot reaction with SAP (Shrimp

Table 2 SNPs in LD with targeted SNP (threshold for LD is $r^{2}>0.8$ )

\begin{tabular}{|c|c|c|c|}
\hline Targeted SNP & SNPs in LD & Targeted SNP & SNPs in LD \\
\hline \multirow[t]{3}{*}{ rs875119 } & rs1791185 & rs62093482 & rs62093482 \\
\hline & rs875120 & rs 1791228 & rs1791228 \\
\hline & rs875119 & rs4799586 & rs12458967 \\
\hline rs3764478 & rs3764478 & & rs 13381522 \\
\hline rs1800458 & rs1800458 & & rs17740990 \\
\hline \multirow[t]{2}{*}{ rs72922947 } & rs72922938 & & rs1791185 \\
\hline & rs72922947 & & rs1791190 \\
\hline \multirow[t]{13}{*}{ rs7235277 } & rs 1791200 & & rs1791196 \\
\hline & rs723744 & & rs1791206 \\
\hline & rs3794884 & & rs3764477 \\
\hline & rs1080093 & & rs4799580 \\
\hline & rs 1667254 & & rs4799586 \\
\hline & rs7235277 & & rs4799587 \\
\hline & rs 3764476 & & rs875119 \\
\hline & rs1791198 & & rs9948445 \\
\hline & rs3764479 & & rs4799586 \\
\hline & rs1791199 & & \\
\hline & rs1611949 & & \\
\hline & rs1667255 & & \\
\hline & rs7235277 & & \\
\hline
\end{tabular}

SNP single nucleotide polymorphism, $L D$ linkage disequilibrium
Alkaline Phosphatase, USB Corporation), as recommended by the manufacturer. The SNaPshot products were loaded in an ABI-PRISM $3130 \mathrm{XL}$ genetic analyzer (Applied Biosystems) and genotyped with GeneMapper

4.0 software (Applied Biosystems). To confirm uncertain genotypes, some individuals were additionally genotyped by sequencing. Sequencing was performed using the Big Dye Terminator Cycle Sequencing v1.1 Ready Reaction (Applied Biosystems), according to the manufacturer's instructions, and samples loaded on an ABI-PRISM 3130 XL genetic analyzer (Applied Biosystems).

Haplotypes were constructed in informative families and inferred, using SNPator software, whenever the phase could not be directly determined [19].

\section{Statistical and SNPs Analysis}

Allelic frequencies were compared in early vs. late cases using Haploview 4.1 with all parameters set at the default values. In order to correct for multiple comparisons, tests were performed using 10,000 permutations [17].

Since we included in the analysis several members of the same family, each patient was Bnested ${ }^{\wedge}$ in his/her fam- ily. To account for non-independency of AO between members of the same family, we performed a weighted analysis using generalized estimating equations (GEEs) [20]. Therefore, we assessed any simultaneous association of the different variants with AO (as the dependent variable), using the most common genotype as the reference

category. The unstandardized coefficient (B) corresponds to the mean AO variation observed in the individuals carrying a specific genotype when compared with the reference category.

To compare haplotypes frequency in FAP samples and controls and in parent-offspring pairs, a chi-square test was used and odds ratios (OR) estimated, with $95 \%$ confidence intervals (CI). These analyses were performed using IBM SPSS Statistics software (v.20; Armonk, NY, USA).

The software is-rSNP (for in silico regulatory SNP detection) was used to explore putative changes in the transcription factors binding capacity due to the variants present. We set the significance level for the is-rSNP analysis at 0.05 and the JASPAR database was used as reference [21].

\section{Results}

We studied 8 SNPs at the TTR locus in a sample of 910 Portuguese individuals, comprising 589 Val30Met carriers,

in order to search for genetic modifiers closely linked to the TTR locus. 


\section{Construction of Haplotypes}

We surveyed the genetic variation across a $57 \mathrm{~kb}$ region spanning the TTR locus and the haplotypes were reconstructed, using eight SNPs: rs875119, rs3764478, rs1800458, rs72922947, rs7235277, rs62093482, rs1791228, and rs4799586 (Fig. 1).

The estimated haplotype frequencies in FAP patients (just one patient per family) and controls are shown in Table 3 . The most common was haplotype A, both in FAP patients (68.7\%) and the control group (47.6\%), followed by haplotype D. Haplotype A, however, was relatively more frequent ( $p$ $<0.001$ ) in mutation carriers than in controls (haplotype A against all other haplotypes); there were no significant differences in the relative frequencies of other haplotypes between patients and controls.

\section{Parent-Offspring Transmissions}

When looking at haplotypes' transmission, haplotype D was preferentially transmitted by the non-carrier parent ( $p$ $<0.001)$. On the other hand, haplotype A was strongly associated with Val30Met: in 97 (96\%) of the 101 transmissions, haplotype A cosegregated with the mutation, while haplotype D corresponded to the other four families. Presence of haplotypes A and D did not determine anticipation ( $>10$ years) $(p>0.05)$. The sex of the transmitting parent did not show any association with the haplotypes transmitted when comparing early to late-onset patients. There was, nevertheless, a trend towards early AO in the offspring when the carrier parent transmitted haplotype D s compared to other haplotypes altogether (except haplotype A) $(p=0.055)$. Importantly, we found that haplotype $\mathrm{C}$ is always transmitted by the noncarrier parent.

\section{Very Early vs. Late-Onset Cases}

When we analyzed the haplotype distribution between very early ( $\leq 30$ years) and late-onset ( $\geq 50$ years) patients, haplotype A was clearly the most frequent in both groups (over 50\%); but, importantly, haplotype $\mathrm{C}$ was more frequent in very early than in late-onset patients $(p=0.009)$ (Fig. 2).

\section{Analysis of Allele Frequencies}

Comparing allelic frequencies of each SNP between very early ( $\leq 30$ years) and late onset ( $\geq 50$ years), we found an association of the A allele of rs72922947 with very early onset $(p=0.009)$; this remained significant after permutation-based correction (Table 4). These results remained significant when we expanded this group to earlyonset cases ( $\leq 40$ years); however, this did not re- main significant after permutation-based correction $(10,000$ permutations) (not shown).

Also, the rs72922947 A allele frequency in the control sample did not differ significantly from that found in the 1000 Genomes project for Utah Residents (CEPH) with Northern and Western European ancestry $(p>0.05)$. On the other hand, there was a slightly increased frequency of the A allele in very early-onset patients, when compared with 1000 Genomes database (0.033 and 0.012 , respec- tively) (Table 4), although the differences were not signif- icant ( $p$ $>0.05)$.

\section{Analysis of Genotype Frequencies}

In a group of 429 patients and in accordance with the results found in the analysis of alleles, the GA genotype of
Fig. 1 Structure of the estimated haplotypes and position of the SNPs relatively to $T T R$. The eight single nucleotide polymorphism (SNP) used for the haplotypes constructed and their location across a $57 \mathrm{~kb}$ region spanning the TTR locus

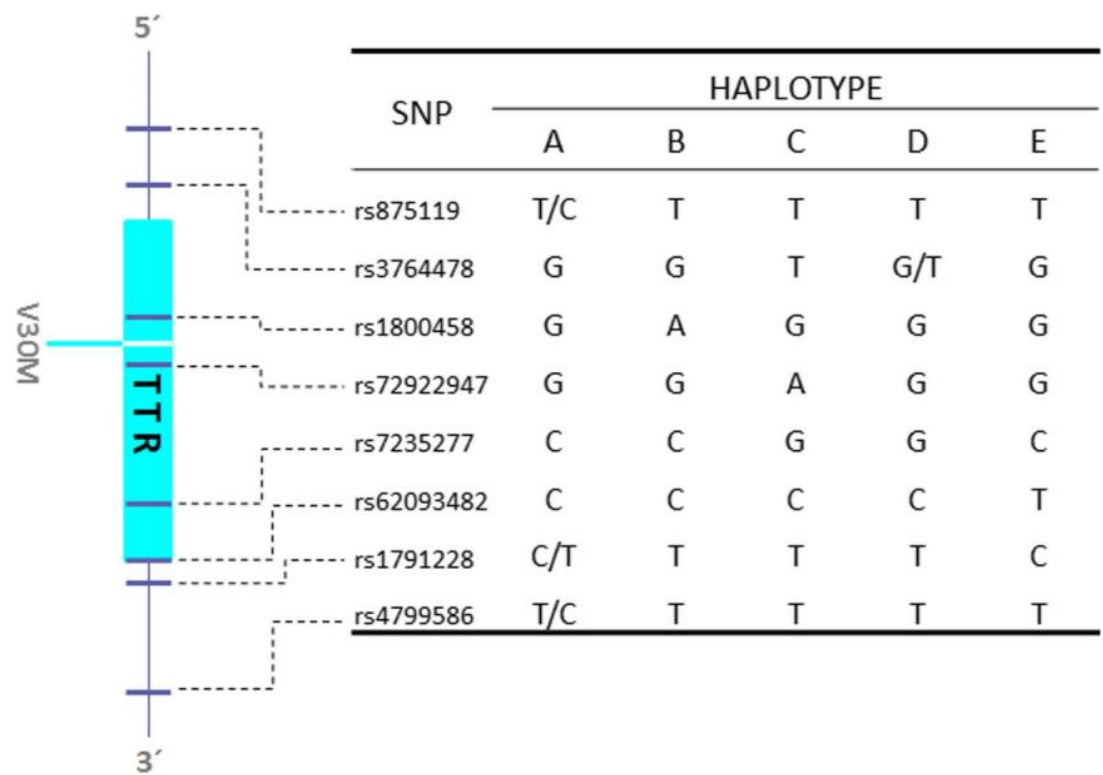


Table 3 Haplotype frequency in Val30Metcarriers (just one patient per family) and unrelated controls

\begin{tabular}{ccc}
\hline Haplotype & $\begin{array}{l}\text { Val30Met }(n=155) \\
(\%)\end{array}$ & $\begin{array}{l}\text { Controls }(n=189) \\
(\%)\end{array}$ \\
\hline & & $180(47.6)$ \\
$\mathrm{A}^{\mathrm{a}}$ & $213(68.7)$ & \\
$\mathrm{B}$ & $10(3.2)$ & $23(6.1)$ \\
$\mathrm{C}$ & $4(1.3)$ & $4(1.1)$ \\
$\mathrm{D}$ & $77(24.8)$ & $152(40.2)$ \\
$\mathrm{E}$ & $6(2)$ & $14(3.7)$ \\
$\mathrm{O}$ & $0(0)$ & $5(1.3)$
\end{tabular}

${ }^{a}$ Haplotype A was relatively more frequent $(p<0.001)$ in mutation carriers than in controls

rs72922947 was associated with a significant decrease in AO ( $p=0.014)$, a mean difference of 8.58 years, when compared with the GG genotype (the reference class) (B, $-8.58 ; 95 \% \mathrm{CI}$, -15.46 to -1.70 ) (Fig. 3).

Functional Impact: In Silico Analysis

We performed an in silico analysis using is-rSNP, with particular attention to rs72922947. The is-rSNP algorithm reported that the DNA binding affinity of three transcription factors is significantly affected by this SNP (LM58, $p=0.005$; LM56, $p=0.01$; and LM233, $p=0.023$ ).

Additionally, we have also analyzed SNPs in linkage disequilibrium with rs 72922947 , and found that rs 72922938 may alter the TP53 $(p=0.001)$ binding site in the TTR gene (Fig. 4).

\section{Discussion}

Variability in AO in FAPATTRV30M has been a most intriguing feature and the object of some previous research. Our strategy now was to identify genetic modifiers within the TTR (FAP) locus, or closely linked to it, that might modulate AO, using a haplotype study. We found a haplotype (and a
Table 4 Allelic analysis comparing very early- $(\leq 30)$ vs. late-onset $(\geq 50$ years) patients

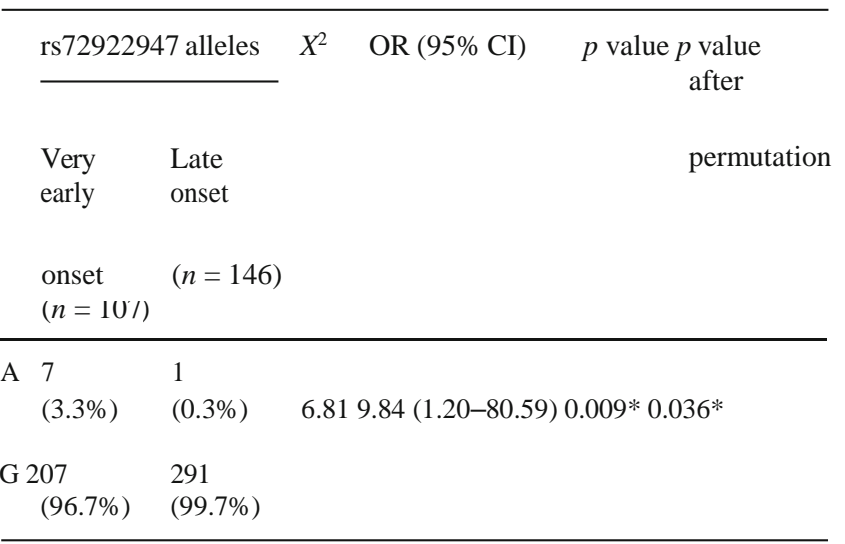

$O R$ odds ratio, $C I$ confidence interval

$*_{p}<0.05$

specific variant within it) that confers an increased risk to Bvery early^-onset patients, which may partially explain the earlier onset in the offspring, when transmitted by the noncarrier parent (trans effect).

Unraveling TTR Variants Associated with AO Modulation

We confirmed a possible trans effect on AO, exerted by haplotype $\mathrm{C}$ (more frequent in very early and early than in late-onset cases). All patients with this haplotype received it from the non-carrier parent and the haplotype A from the affected parent, confirming the hypothesis of a trans- acting factor within the TTR locus associated with AO variability.

The rs 72922947 (c. $200+795 \mathrm{G}>\mathrm{A}$ ), located within intron 2 of the TTR gene, is represented by the minor allele $\mathrm{A}$ in haplotype $\mathrm{C}$, distinguishing this from the other haplotypes that carried the common allele G. Both allelic and genotypic analysis showed a significant association with an earlier AO. Accordingly, carriers of the GA genotype tend to show a decrease of $\mathrm{AO}$, on average of nearly 9 years. Furthermore, allele $\mathrm{A}$ is a risk factor strongly associated with (at least) very early onset ( $\leq 30$ years).

In addition, our results show that the A allele is more frequent in the very early-onset cases than expected
Fig. 2 Haplotype distribution in Val30Met carriers in very early ( $\leq 30$ years) vs. late-onset patients $(\geq 50$ years $)$

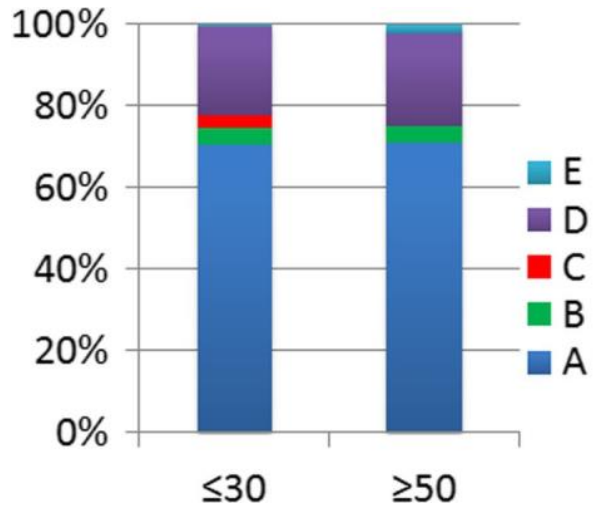

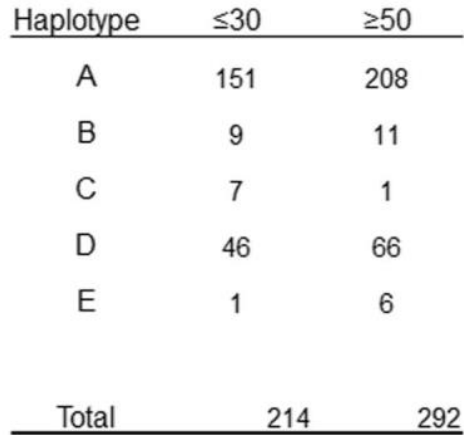




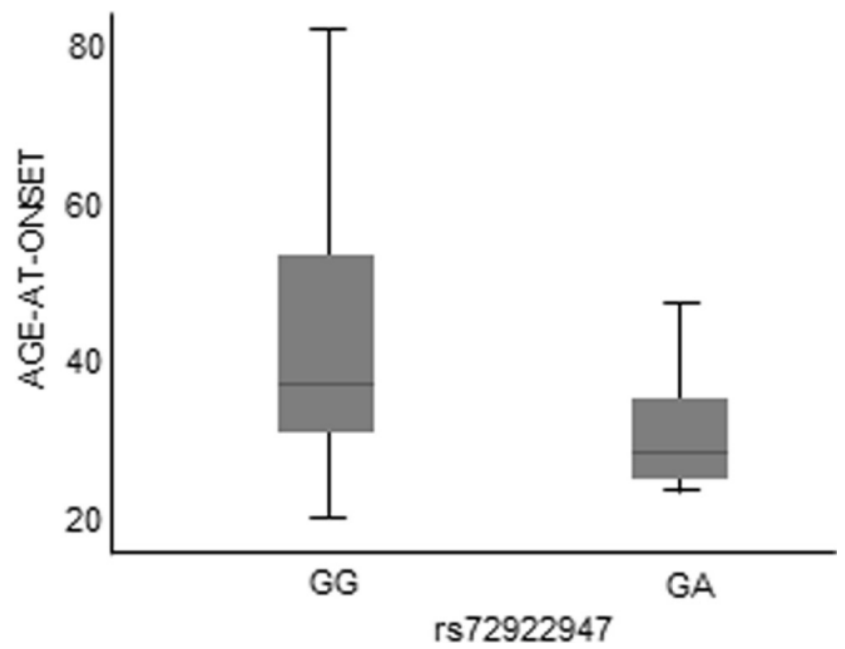

Fig. 3 Genotypic variation in rs72922947 according to age at onset. Boxplots showing the association of rs 72922947 with age at onset, by genotype ( $\mathrm{GG}$ and $\mathrm{GA}$ ); none of our subjects carried the rare homozygous genotype (AA)

according to the frequency in Portuguese controls (similar to those on the 1000 Genomes project data for Utah Residents (CEPH) with Northern and Western European ancestry). This reinforces the findings that the minor al- lele A of rs72922947 was overrepresented in our FAP cohort.
In Silico Analysis

The in silico analysis of TFBS (transcription factors binding sites) allowed us to estimate the probability of modulation by this SNP of the expression/activity of TTR that could drive the clinical presentation of the disease. Few studies have investigated the role of non-coding SNPs in the TTR gene and its possible functional consequences. Polimanti et al. identified 59 non-coding variants that may have a functional impact on the TTR gene, including rs 72922947 ; however, further studies were required to understand the role of this variant [22]. Three transcription factors binding sites (LM56, LM58, and LM233) were predicted to be disrupted by rs72922947. The minor allele (A) was predicted to have less affinity for all these transcription factors; however, there are scarce data about the role of these TFBS. Additionally, we verified that rs72922938, a SNP that is in linkage disequilibrium with rs72922947 may alter the TP53 binding site. TP53 has been reported as a genetic modifier of AO in several cancers, as well as in Huntington disease (HD) in which only $30-70 \%$ of the variance in $\mathrm{AO}$ may be explained by the CAG repeat size alone [23].

These results will enable us to test new hypotheses. Whether this rs72922947 is itself the functional polymorphism responsible for exerting a direct effect on the TTR gene
Fig. 4 The sequence logos of the three TFBS potentially disrupted by rs72922947 and rs72922938. Transcription factor (TF) binding sites (TFBS) potentially disrupted by the TTR single nucleotide polymorphisms (SNPs). The logo plots created by is-rSNP [21] were matched manually to the gene sequence surrounding the marked SNP, as shown below by the transcription factor binding profile (the SNP alleles are shown with the common allele listed first; SNP alleles that differ from taller letters in the binding profile have the largest potential impact)

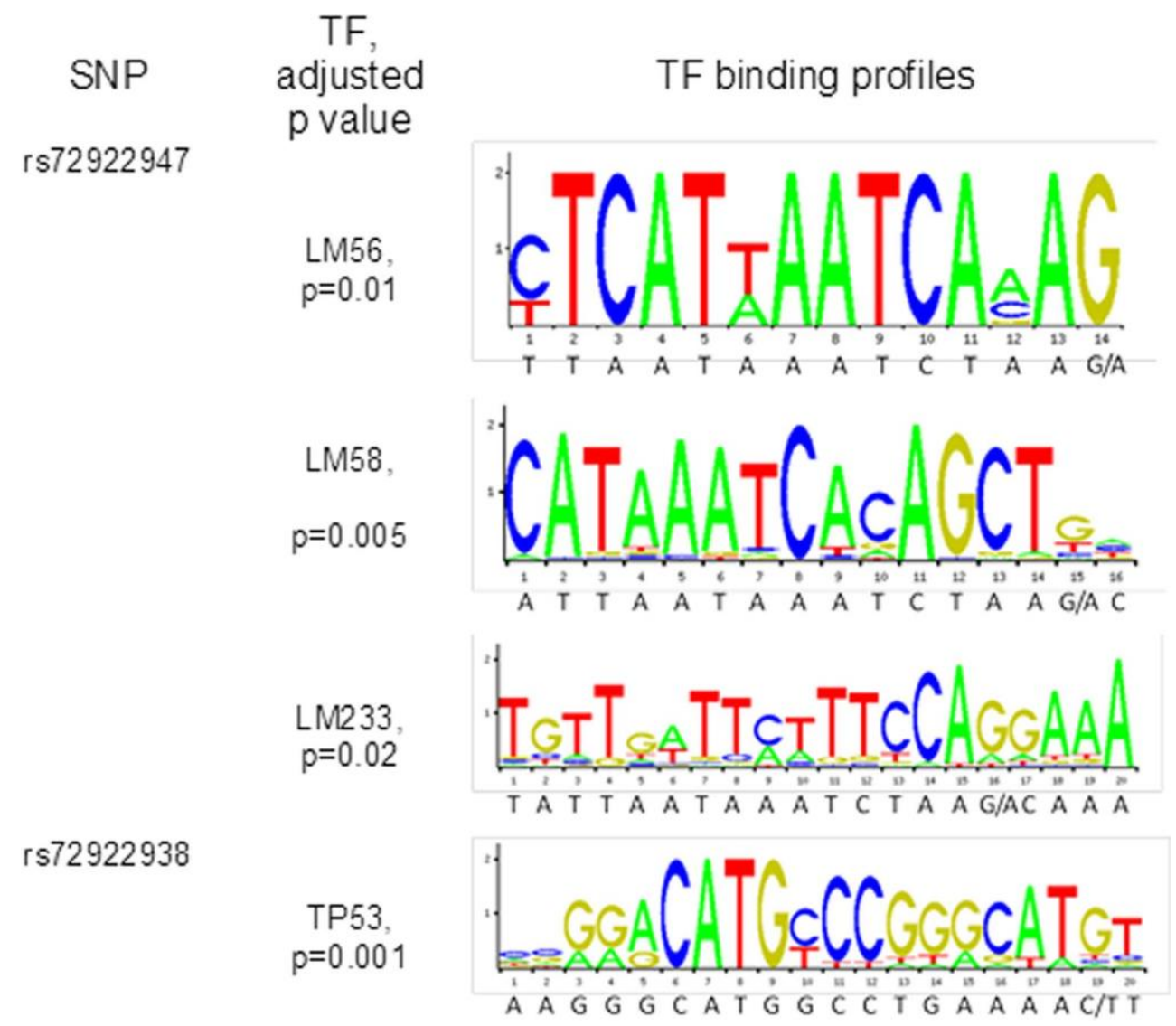


expression, or if it is only in linkage disequilibrium with the functional SNP is still an open question.

These hypotheses have also been assessed in other neurodegenerative diseases like HD, where common genetic variants near the mutation site were explored as possibly associated with AO, although no significant results were found [24].

In a previous study, Soares et al. focused mainly on comparing extended haplotypes in 18 late-onset (onset $\geq 50$ years) and 19 Bclassic-onset $\wedge$ patients ( $<40$ years), vs. controls [15]. It was suggested a possible modulatory effect on AO, exerted by a locus within or closely linked to the interval between microsatellites D18S457 and D18S456 (but not by the immediately 5' and 3' TTR flanking sequences), and associated with the non-carrier chromosome (i.e., a trans-acting effect more frequent in late than in early-onset cases). This failed, however, to reach significance, possibly due to the effect being too small and/or to small sample size. Likewise, the variation within the same family and, particularly, within and among generations was not taken into account. These results still lack validation. Our study showed a similar effect but now within the TTR (FAP) locus itself.

Recently, Sikora et al. found a nearly significant association between wild-type TTR amyloidosis and rs1800458; a variant in this SNP was predicted to be protector despite the acknowledgment that their results are insufficient to prove this [25]. In our study, this variant showed no statistically significant results that could indicate a putative association with AO. On the other hand, these authors confirmed that this missense variant is non-pathogenic.

In a sample of Swedish patients, which is characterized by low penetrance and high AO, all four TTR exons and flanking regions were sequenced [15]. In that study, rs62093482 was the only SNP which effect was significant after correction for multiple testing; since this is located in the 3' UTR, it was suggested that it could affect TTR expression levels by modifying microRNA binding. Later functional studies, however, did not confirm this [26]. Thus, as reported in this paper, the rs62093482 does not seem to act as a modifier of AO variability.

\section{Conclusions}

Haplotype A, followed by haplotype D, is overrepresented in chromosomes with the Val30Met mutation in the Portuguese population. Haplotype $\mathrm{C}$ is transmitted by the non-carrier parent as described in the Results.

A trans-acting effect modulates the phenotypic expression of FAP ATTRV30M, with a sequence variant (A) and genotype (GA) predisposing to early onset. Since we found in a previous study [9] that parents with an $\mathrm{AO} \leq 30$ years also have a higher risk of also having offspring with $\mathrm{AO} \leq 30$ years, the study of these very early-onset patients helped us to define higher-risk groups where it is important to look for genetic modifiers.

Understanding some of the mechanisms that may influence AO may help predicting AO, which will be of importance for genetic counseling and the follow-up of presymptomatic carriers. These results may prove useful for therapeutic strategies, as the identification of risk genetic modifiers associated with AO (early vs. late onset, non-penetrance) will have important clinical implications.

Acknowledgements The authors thank all the patients and families for participating in this study and Vanessa Costa (from Unidade Corino de Andrade (UCA), Centro Hospitalar do Porto (CHP)) for all the help in data collection. This work was supported by grants of Fundação para a Ciência e Tecnologia,FCT [PTDC/SAU-GMG/100240/2008 and PEsT], cofunded by ERDF and COMPETE; and by Financiamento Plurianual de Unidades de Investigação (FCT).

This is a post-peer-review, pre-copyedit version of an article published in Molecular Neurobiology. The final authenticated version is available online at: http://dx.doi.org/10.1007/s12035-017-0593-4.

Compliance with Ethical Standards

Conflict of Interest M.A.F. and D. S. have received research support from a FCT fellowship (SFRH/BD/101352/2014 and SFRH/BD/91160/ 2012, respectively). T. C.'s institution has received support from FoldRx Pharmaceuticals, which was acquired by Pfizer Inc. in October 2010; T. C. has served on the scientific advisory board of Pfizer Inc. and received funding from Pfizer Inc. for scientific meeting expenses (travel, accommodations, and registration). She currently serves on the THAOS (natural history disease registry) scientific advisory board. J. S., I. A., A. S., and C. L. report no disclosures. 
1. Andrade C (1952) A peculiar form of peripheral neuropathy; familiar atypical generalized amyloidosis with special involvement of the peripheral nerves. Brain 75(3):408-427

2. Benson MD (2012) Pathogenesis of transthyretin amyloidosis. Amyloid 19(Suppl 1):14-15. doi:10.3109/13506129.2012.668501

3. Sousa A, Coelho T, Barros J, Sequeiros J (1995) Genetic epidemiology of familial amyloidotic polyneuropathy (FAP)-type I in Povoa do Varzim and Vila do Conde (north of Portugal). Am J Med Genet 60(6):512-521. doi:10.1002/ajmg.1320600606

4. Sequeiros J, Saraiva MJ (1987) Onset in the seventh decade and lack of symptoms in heterozygotes for the TTRMet30 mutation in hereditary amyloid neuropathy-type I (Portuguese, Andrade). Am J Med Genet 27(2):345-357. doi:10.1002/ajmg.1320270213

5. Sousa A, Andersson R, Drugge U, Holmgren G, Sandgren O (1993) Familial amyloidotic polyneuropathy in Sweden: geographical distribution, age of onset, and prevalence. Hum Hered 43(5): 288-294

6. Saporta MA, Zaros C, Cruz MW, Andre C, Misrahi M, Bonaiti- Pellie C, Plante-Bordeneuve V (2009) Penetrance estimation of TTR familial amyloid polyneuropathy (type I) in Brazilian families. Eur J Neurol 16(3):337-341. doi:10.1111/j.1468-1331.2008. 02429.x 7. Munar-Ques M, Saraiva MJ, Viader-Farre C, Zabay-Becerril JM, Mulet-Ferrer J (2005) Genetic epidemiology of familial amyloid polyneuropathy in the Balearic Islands (Spain). Amyloid 12(1): 54-61

8. Ikeda S, Hanyu N, Hongo M, Yoshioka J, Oguchi H, Yanagisawa N, Kobayashi T, Tsukagoshi H et al (1987) Hereditary generalized amyloidosis with polyneuropathy. Clinicopathological study of 65 Japanese patients. Brain 110(Pt 2):315-337

9. Lemos C, Coelho T, Alves-Ferreira M, Martins-da-Silva A, Sequeiros J, Mendonca D, Sousa A (2014) Overcoming artefact: anticipation in 284 Portuguese kindreds with familial amyloid polyneuropathy (FAP) ATTRV30M. J Neurol Neurosurg Psychiatry 85(3):326-330. doi:10.1136/jnnp-2013-305383

10. Sousa A (1995) A Variabilidade Fenotípica da Polineuropatia Amiloidótica Familiar: um estudo de Genética Quantitativa em Portugal e na Suécia. ICBAS, Univ Porto, Porto

11. Coelho T, Sousa A, Lourenco E, Ramalheira J (1994) A study of 159 Portuguese patients with familial amyloidotic polyneuropathy (FAP) whose parents were both unaffected. J Med Genet 31(4): 293-299

12. Zaros C, Genin E, Hellman U, Saporta MA, Languille L, Wadington-Cruz M, Suhr O, Misrahi M et al (2008) On the origin of the transthyretin Val30Met familial amyloid polyneuropathy. Ann Hum Genet 72(Pt 4):478-484

13. Yoshioka K, Furuya H, Sasaki H, Saraiva MJ, Costa PP, Sakaki Y (1989) Haplotype analysis of familial amyloidotic polyneuropathy. Evidence for multiple origins of the Val-Met mutation most common to the disease. Hum Genet 82(1):9-13

14. Almeida MR, Aoyama-Oishi N, Sakaki Y, Holmgren G, Drugge U, Ferlini A, Salvi F, Munar-Quès M, et al. (1995) Haplotype analysis of common transthyretin mutations. HumGenet

15. Soares ML, Coelho T, Sousa A, Holmgren G, Saraiva MJ, Kastner DL, Buxbaum JN (2004) Haplotypes and DNA sequence variation within and surrounding the transthyretin gene: genotype-phenotype correlations in familial amyloid polyneuropathy (V30M) in Portugal and Sweden. Eur J Hum Genet 12(3):225-237. doi:10. 1038/sj.ejhg.5201095

16. Miller SA, Dykes DD, Polesky HF (1988) A simple salting out procedure for extracting DNA from human nucleated cells. Nucleic Acids Res 16(3):1215

17. Barrett JC, Fry B, Maller J, Daly MJ (2005) Haploview: analysis and visualization of LD and haplotype maps. Bioinformatics 21(2): 263-265. doi:10.1093/bioinformatics/bth457

18. Ii S, Sommer SS (1993) The high frequency of TTR M30 in familial amyloidotic polyneuropathy is not due to a founder effect. Hum Mol Genet 2(8):1303-1305

19. Morcillo-Suarez C, Alegre J, Sangros R, Gazave E, de Cid R, Milne R, Amigo J, Ferrer-Admetlla A et al (2008) SNP analysis to results (SNPator): a web-based environment oriented to statistical genomics analyses upon SNP data. Bioinformatics 24(14):1643-1644. doi:10.1093/bioinformatics/btn241

20. Zeger SL, Liang KY (1986) Longitudinal data analysis for discrete and continuous outcomes. Biometrics 42(1):121-130

21. Macintyre G, Bailey J, Haviv I, Kowalczyk A (2010) is-rSNP: a novel technique for in silico regulatory SNP detection. Bioinformatics 26(18):i524-i530. doi:10.1093/bioinformatics/ btq378

22. Polimanti R, Di Girolamo M, Manfellotto D, Fuciarelli M (2014) In silico analysis of TTR gene (coding and non-coding regions, and interactive network) and its implications in transthyretin-related amyloidosis. Amyloid 21(3):154-162. doi:10.3109/13506129. 2014.900487

23. Chattopadhyay B, Baksi K, Mukhopadhyay S, Bhattacharyya NP (2005) Modulation of age at onset of Huntington disease patients by variations in TP53 and human caspase activated DNase (hCAD) genes. Neurosci Lett 374(2):81-86

24. Lee JM, Gillis T, Mysore JS, Ramos EM, Myers RH, Hayden MR, Morrison PJ, Nance M et al (2012) Common SNP-based haplotype analysis of the 4p16.3 Huntington disease gene region. Am J Hum Genet 90(3):434-444

25. Sikora JL, Logue MW, Chan GG, Spencer BH, Prokaeva TB, Baldwin CT, Seldin DC, Connors LH (2015) Genetic variation of the transthyretin gene in wild-type transthyretin amyloidosis (ATTRwt). Hum Genet 134(1):111-121. doi:10.1007/s00439- 014-1499-0

26. Norgren N, Hellman U, Ericzon BG, Olsson M, Suhr OB (2012) Allele specific expression of the transthyretin gene in Swedish patients with hereditary transthyretin amyloidosis (ATTR V30M) is similar between the two alleles. PLoS One 7(11):e49981. doi:10.

1371/journal.pone.0049981 